\title{
La importancia de las nuevas tecnologías en la democracia participativa. El caso de la Iniciativa Ciudadana Europea ${ }^{1}$
}

\section{The value of new technology in participatory democracy. The case of the European Citizens' Initiative}

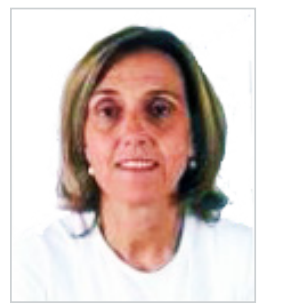

Maria Manuela Magalhães Silva. Doctora en Derecho. Profesora Asociada, licenciada por la Facultad de Derecho de la Universidad de Coimbra, Portugal. Máster en Relaciones Internacionales y Doctora en Derecho-Ciencias Jurídico-Políticas. Miembro e investigadora principal del Instituto Jurídico Portucalense (IJP), acreditada por la Fundación para la Ciencia y Tecnología (Fundação para a Ciência e a Tecnologia, (FCT)), integrada en la IG: Dimensión derechos humanos. Investigadora asociada al Centro Europeo de Derecho Económico, Financiero y Tributario-CIDEEFF, Facultad de Derecho de Lisboa e integrada en la IG "Globalización, Integración Económica y Desarrollo: European and Lusophone Perspectives”; acreditada por la FCT con producción científica en las áreas de Derecho Constitucional, Derechos Fundamentales y Derecho de la Unión Europea.

Universidade Portucalense, Porto, Portugal

mmdmms@upt.pt

ORCID: 0000-0003-4261-7271

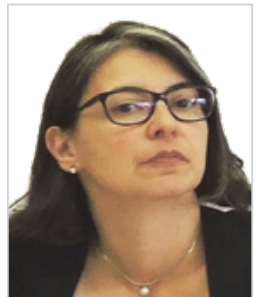

Dora Resende Alves. Profesora Auxiliar del Departamento de Derecho de la Universidade Portucalense (UPT), Portugal, con experiencia en unidades curriculares en el ámbito del Derecho de la Unión Europea, Derecho Constitucional e Historia del Derecho. Doctora por la Universidad de Vigo (España) con una tesis sobre el derecho de la competencia de la Unión Europea. Máster en Derecho, área de Integración Europea, por la Facultad de Derecho de la Universidad de Coimbra. Profesora del Colegio de Abogados del Centro Regional de Oporto. Investigadora permanente del Instituto Jurídico Portucalense, Centro de Investigación de la Universidad de Portucalense. Redactora adjunta de la Revista Jurídica Portucalense.

Universidade Portucalense, Porto, Portugal

dra@upt.pt

ORCID: 0000-0003-4720-1400

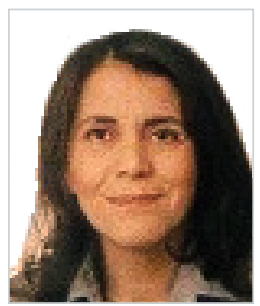

María João Ferreira. Profesora Asociada del Departamento de Ciencia y Tecnología de la Universidade Portucalense, (UPT) Portugal. Doctora en Informática en 2003 por la Universidad de Manchester. Es investigadora del REMIT de la Universidade Portucalense y del ISTTOS-Centro Algoritmi de la Universidad del Minho. Su investigación se centra en los Sistemas de Información y la transformación digital en las Organizaciones. Es coautora de varias de publicaciones científicas, capítulos de libros y revistas y ha participado en varias conferencias internacionales como miembro del comité de programa, comité organizador y miembro del consejo local. También pertenece al Consejo Editorial de IJADS y de IJAS.

Universidade Portucalense, Porto, Portugal

mjoao@upt.pt

ORCID: 0000-0003-4274-8845

1 Titulo de la Comunicación presentada en el I Congreso Internacional sobre Vulnerabilidad y Cultura Digital, Madrid, España, 18-19 de octubre de 2018

Cómo citar este artículo:

Magalhães Silva, M. M.; Resende Alves, D. y João Ferreira, M. (2019). The value of new technologies in participatory democracy. The case of the European citizens' initiative. Doxa Comunicación, 28, pp. 37-53.

https://doi.org/10.31921/doxacom.n28a02 
Recibido: 18/11/2018 - Aceptado: 17/05/2019

\section{Resumen:}

La participación democrática sigue siendo el camino a seguir por las sociedades que buscan una paz estable mediante el ejercicio de una ciudadanía activa. Desde 2012, el instrumento Iniciativa Ciudadana Europea está presente en el proceso de elaboración de las disposiciones jurídicas y normativas de la Unión Europea. Uno de los aspectos de esta ciudadanía es el de permitir a los ciudadanos que se involucren en la toma de decisiones a través de la participación en el ejercicio del trabajo legislativo. El Tratado de Lisboa introdujo el derecho a la Iniciativa Ciudadana Europea (en ingles, ECI). El objetivo era implicar activamente a los ciudadanos en la toma de decisiones en Europa, ofreciéndoles una forma indirecta de iniciativa legislativa. Las tecnologías de la información (TI) son, en general, la base de las organizaciones modernas, y los cambios en este campo se producen a un ritmo vertiginoso, deteniendo el desarrollo de los modelos tradicionales y obligando a las organizaciones a implementar nuevos modelos, nuevas formas de trabajar y comunicarse. En este contexto, la Comisión Europea, sin ignorar la relevancia y las transformaciones inherentes al uso de las Tecnologías de la Información, ha incluido el uso de estas tecnologías en varios ámbitos, concretamente en la Iniciativa Ciudadana Europea. En esta investigación se analiza en qué medida una plataforma, disponible en el contexto de la Iniciativa Ciudadana Europea, se utiliza en las peticiones y la evolución de su uso hasta la finalización de la revisión en 2018.

\section{Palabras clave:}

Iniciativa Ciudadana Europea; legislación; Unión Europea Tecnología de la Información; participación digital.

\section{Received: 18/11/2018 - Accepted: 17/05/2019}

\section{Abstract:}

Democratic participation still is the way forward for societies that seek permanent peace through the exercise of active citizenship. Since 2012, the European Citizens' Initiative instrument has emerged in the process of drafting legislative acts of the European Union. One of the facets of this citizenship is to enable citizens to become involved in decision-making through participation in the exercise of legislative initiative. The Lisbon Treaty introduced the right to the ECI. The aim is to actively involve citizens in the European decision-making, giving them an indirect form of legislative initiative. Information technologies (IT) are the essence of up-to-date organizations in general, and changes in this field are occurring at an uncontrollable pace, interrupting traditional models and forcing organizations to implement new models, new ways of working and communicating. In this context, the European Commission, not ignoring the relevance and transformations inherent in the use of IT, has included its use in several areas, namely in the European Citizens' Initiative. In this research is analysed how much a platform available in the European Citizens' Initiative context is used in petitions and the evolution of this same use until the completion of the review in 2018.

\section{Keywords:}

European Citizens Initiative; legislation; European Union, Information Technology, digital participation.

\section{Introducción}

La participación democrática es el camino a seguir por las sociedades que buscan la paz de forma estable a través del ejercicio de una ciudadanía activa. Desde 2012, la herramienta Iniciativa Ciudadana Europea está presente en el proceso de elaboración de las disposiciones jurídicas y normativas de la Unión Europea.

La ciudadanía europea proporciona a los ciudadanos europeos importantes derechos y la posibilidad de una participación democrática y activa en el proceso de toma de decisiones de la Unión Europea (EU, en inglés). Una de las características de esta ciudadanía es que permite a los ciudadanos intervenir en la toma de decisiones a través de su participación en el ejercicio de la iniciativa legislativa. Esto ocurre tanto a nivel nacional, casos de Portugal y España, y también en la Unión Europea. Este es un camino muy prometedor. Los resultados son aún escasos debido, en parte, al escaso tiempo de puesta en funcionamiento de la herramienta pero es un camino importante. Creemos que se convertirá en una forma de empoderar a los ciudadanos para que influyan en la agenda política. 
La UE intenta cada vez más sensibilizar sobre los derechos de la ciudadanía, fomentando la participación cívica de los jóvenes donde el mayor uso de la tecnología puede conducir a un mayor grado de aceptación.

Además, el uso de las nuevas oportunidades creadas por la tecnología ha abierto nuevas perspectivas a la participación política de los ciudadanos, permitiendo que sus aportaciones lleguen al sistema político (Gil de Zúñiga, Huber y Strauß, 2018), principalmente a través del uso de las redes sociales (Marcos García, Alonso Muñoz y Casero-Ripollés, 2017). El objetivo de este trabajo, sin embargo, se centra en la Iniciativa Ciudadana Europea (ECI, en inglés)

En la actualidad, disponemos de nuevas herramientas tecnológicas que facilitan un nuevo ámbito de participación ciudadana, con un gran potencial político (Chadwick, 2017), aunque aún no puede afirmarse que estas sean exitosas a la hora de llevar a cabo un ejercicio individual, legítimo e informado (Aguilera y Casero Ripollés, 2018).

El Tratado de Lisboa estableció el derecho a la Iniciativa Ciudadana Europea. Permite a los ciudadanos de la UE, de al menos siete Estados miembros, invitar a la Comisión Europea a presentar propuestas legislativas en ámbitos de competencia de la Unión Europea como condición legal para su admisibilidad. El objetivo es implicar activamente a los ciudadanos en la toma de decisiones, ofreciéndoles una forma indirecta de iniciativa legislativa. Hasta la fecha, más de 9 millones de ciudadanos europeos han firmado una Iniciativa Ciudadana Europea.

Es sólo después del proceso de registro cuando sus organizadores inician el proceso de recogida de apoyos a la propuesta, la cual necesita del apoyo de un millón de ciudadanos de al menos una cuarta parte de los Estados miembros de la UE. Se trata de una invitación para que la Comisión actúe ante la presentación de propuestas legislativas. Hasta ahora, con cuatro casos muy diferentes que cumplían todos los requisitos, no hay aún ninguna iniciativa plenamente satisfactoria que haya dado lugar a una propuesta legislativa concreta. Sin embargo, es un instrumento de futuro y de gran trascendencia democrática a tener en cuenta, como señala la metodología de análisis crítico documental y legislativo seguida.

La ICE está totalmente operativa pero es posible evaluarla y mejorarla, y su revisión y simplificación está en curso, con el fin de hacerla más fácil de utilizar y más accesible a los ciudadanos.

Las tecnologías de la información (TI) son la esencia de las organizaciones modernas, en general, y los cambios en este campo se producen a un ritmo vertiginoso, deteniendo el desarrollo de los modelos tradicionales y obligando a las organizaciones a implementar nuevos modelos, nuevas formas de trabajar y (Schuelke-Leech, 2018). En este contexto, la Comisión Europea (en inglés, EC) no ignora la relevancia y las transformaciones inherentes al uso de las TI - ente las cuales se incluye Internet con todo su potencial, a saber; el acceso a la información, la comunicación y la colaboración en el trabajo (Murray, 2016)- y ha incluido su utilización en varias áreas. Un ejemplo de ellas es una plataforma desarrollada y puesta al servicio de los ciudadanos la cual permite a estos participar fácil y eficazmente en las políticas que afectan a sus vidas, incluyendo las peticiones promovidas por la propia $\mathrm{CE}$ o por cualquier ciudadano u organización perteneciente a la $\mathrm{CE}$. El resultado es una democratización del instrumento hasta el punto de que la participación ciudadana se vuelve más fácil y agradable.

La integración de las tecnologías para estimular la participación activa de los ciudadanos requiere la comprensión por parte de estos de los medios tecnológicos y de las iniciativas apoyadas por las plataformas on-line puestas a su disposición. En este sentido, las preguntas de investigación que deben responderse son las siguientes: “¿Están los ciudadanos, en el contexto de la iniciativa "ciudadanos europeos", (1) utilizando la plataforma para nuevas peticiones? y (2) i ha aumentado su uso con el paso del tiempo?" Estas preguntas se justifican por la inexistencia en la literatura de trabajos de investigación similares. 
En este contexto, nuestra investigación utiliza la metodología del estudio de caso (Yin, 2009) con el fin de permitir la exploración y comprensión de lo mucho que la plataforma se utiliza en las peticiones y su evolución desde la primera vez que fue usada por parte de las organizaciones y los ciudadanos hasta el presente.

En el caso concreto de la ICE, es evidente que la utilización de los medios que ofrecen las nuevas tecnologías ha facilitado y ampliado su utilización. El resultado es una democratización del instrumento en la medida en que la participación ciudadana se vuelve más fácil y amigable.

\subsection{La construcción democrática europea}

Los valores de libertad, democracia y estado de derecho (Bonavides, 2001; Silva y Alves, 2016) son los lemas en los que se basa la construcción europea, tanto en el pasado como en la actualidad, presentes en la Declaración Schuman de 1950 o documentos del momento que trazan los ejes de acción actuales. Además del artículo 2 del Tratado de la Unión Europea (en inglés, TEU), la Comisión (2001) los consagra diciendo que el Tribunal de Justicia de la Unión Europea garantiza el respeto del estado de derecho, salvaguardado por el mecanismo del artículo 7 del Tratado de la Unión Europea que aborda la vulneración de la democracia, lo que hoy en día está muy en la línea de los problemas políticos de Hungría y Polonia.

Los valores comunes son consecuencia de la historia europea. A pesar de estos valores fundacionales, a menudo se acusa a la UE de un déficit democrático, ya sea en el funcionamiento de las instituciones o en la cuestión del derecho de acceso a los documentos, o incluso en cuestiones actuales relacionadas con la igualdad de género. Consciente de ello, la UE mantiene su apuesta por una mayor transparencia en los procedimientos, el acceso de los ciudadanos a la información y la creación de mejores mecanismos interinstitucionales (Comisión Europea, 2017c).

Es a través de esta construcción democrática, arraigada en la Convención de Filadelfia de 1787, que puede alcanzase una posible homogeneidad de la UE, basada en la ineludible diversidad de los seis países fundadores, - en la actualidad 28 o 27 países, si consideramos la salida del Reino Unido- a través del mecanismo previsto en el artículo 50 del Tratado de la Unión Europea (en inglés, TEU)

Con la seguridad de ser uno de los valores fundacionales hace ya casi 70 años, la caracterización democrática de la Unión Europea sigue hoy abierta al debate, como mantiene Moury (2016), en aspectos tales como la transparencia en la toma de decisiones, el control de las instituciones o la participación de los ciudadanos en la legitimidad de las dos anteriores. Por ello, no debe ponerse en duda que la Unión Europea es, con toda seguridad, uno de los lugares del mundo donde la democracia y los derechos fundamentales están protegidos de forma muy eficaz (Comisión Europea, 2003). Ya son 60 años de paz en un mundo en el que existen 60 conflictos militares, aunque ninguna de estas guerras se ha producido en el territorio de la Unión, tal y como puso de relieve el Presidente de la Comisión Europea, Jean-Claude Juncker en su discurso pronunciado el 25 de marzo de 2017 con motivo del 60 aniversario de los Tratados de Roma que dieron origen a la Comunidad Europea de la Energía Atómica (Euratom) y a la Comunidad Económica Europea (CEE).

En todos los ámbitos, en todas sus actuaciones, y en sus instrumentos, la UE promueve el valor universal de la democracia y la Comisión (2016) ya está pensando en la Agenda 2030. Las instituciones (Comité Europeo de las Regiones, 2015) son conscientes de estos esfuerzos y ello a pesar de tener su propia geometría de poderes, con frenos y contrapesos que no son sino una traducción de estas preocupaciones así como de las diversas reformas sufridas por las instituciones de la 
UE (Pais, 2010). Y es a través de la educación y la formación en los valores comunes y los principios generales de derecho establecidos en los artículos 1 a 3 del TUE que el camino de la UE debe continuarse con el objetivo final de que los participantes adquieran los conocimientos, las aptitudes y las actitudes necesarias para una ciudadanía activa (Consejo, 2017) y democrática. Al garantizar una educación de calidad para todos, en todos los niveles educativos, la política educativa viene a desempeñar un papel de primer orden. Estas competencias sociales, cívicas e interculturales reforzarán, reafirmarán y promoverán los valores democráticos de la UE.

Según la Comisión Europea (2017d), el funcionamiento de la Unión se basa en la democracia representativa, lo que implica transparencia y una cultura política basada en la accesibilidad y la responsabilidad, apoyada por un sistema electoral eficaz y un electorado informado y participativo. A raíz de esta idea, la importancia del mecanismo de la Iniciativa Ciudadana Europea, tal como se conoce fue potenciado. Este logro desempeña un papel importante a la hora de acercar a los ciudadanos al proceso legislativo, como es el caso del Parlamento Europeo (2017a).

\section{En el sistema nacional portugués}

Una de las características de la democracia es la de posibilitar que los ciudadanos se involucren en los procesos de toma de decisiones a través de su participación en el ejercicio de la iniciativa legislativa. Sin ser la única forma de democracia participativa (Maior, 1998), es hoy por hoy un instrumento de ayuda. Ocurre así a nivel nacional en varios países como Portugal, Brasil o España, y también a nivel de la Unión Europea.

En Brasil, en cuatro ocasiones se han aprobado leyes populares como la ley en casos de violencia que movilizó a la opinión pública. Fue la primera vez que su uso dio lugar a la Ley 8.930 de 09/07/1994. Asimismo, en España, la Ley Orgánica 3/1984, de 26 de marzo, reguladora de la iniciativa legislativa popular (consolidada) con las últimas modificaciones introducidas en 2015, garantiza a los ciudadanos el poder de iniciativa legislativa, derecho previsto en el artículo 87.3 de la Constitución Española de 1978.

Este mecanismo no es el único derecho político de inmersión en la democracia (Comisión Europea, 2017a), pero es una herramienta muy prometedora que se ha conseguido de forma clara y fácil de lograr. Los resultados de su utilización son escasos, quizás en consonancia con la juventud del instrumento, pero es un camino importante aunque sólo sea por las aportaciones añadidas. El objetivo es capacitar a los ciudadanos para que puedan influir en la agenda política. El Comité Europeo de las Regiones (2018a) destaca, además, que la diversidad de las estructuras subnacionales de los Estados miembros constituyen un elemento esencial del patrimonio político y cultural de la UE y un punto de referencia clave para el desarrollo de una ciudadanía activa basada en los derechos de ciudadanía de la UE.

En estos términos legales, el proceso legislativo ordinario y parlamentario en la República Portuguesa posibilita que un grupo de votantes pueda presentar un proyecto de ley para aprobación en la Asamblea de la República. Son precisas veinte mil firmas de ciudadanos con derecho a voto que apoyen la idea de ley que se quiere discutir; después se remite al Parlamento donde, tras los trámites del proceso legislativo, puede finalmente ser aprobada. Las firmas pueden recogerse a través de una plataforma informática proporcionada por la propia Asamblea de la República, siendo un recurso que ofrece en, la actualidad, gran potencial de éxito a la iniciativa toda vez que puede captar el interés de las capas más jóvenes de la población. 
Como ejemplo concreto, en 2018 se llevó a cabo una recogida de firmas para proponer la consideración del tiempo total de servicio docente en la enseñanza².

En este sentido, la Unión Europea también reconoció posteriormente esta iniciativa y la presentó.

\section{En la Unión Europea}

Los ciudadanos de la Unión Europea son los verdaderos artífices de la Unión Europea (Comisión Europea, 2002).

En conjunto, la ciudadanía europea proporciona a los ciudadanos europeos varios derechos enunciados en los tratados y en la Carta de los Derechos Fundamentales, y facilita más de una forma de intervenir en la vida política democrática. Las cuestiones sobre el ejercicio participativo, el reconocimiento de la ciudadanía y la participación activa en la vida democrática de la Unión figuran entre las prioridades definidas por la UE que considera este ámbito esencial en sus acciones (Comisión Europea, 2017f). Los estudios citados de la Comisión Europea muestran, además, que los europeos son cada vez más conscientes de su papel como ciudadanos de la Unión y que el porcentaje de europeos que quieren saber más sobre sus derechos es cada vez mayor. Los europeos son mayoritariamente conscientes de que son ciudadanos de la UE, pero no siempre conocen los derechos que se derivan de este hecho. La ciudadanía de la Unión Europea posee importantes derechos y libertades, así como la posibilidad de una participación democrática y activa en el proceso de toma de decisiones de la UE, y sus ciudadanos son importantes a la hora de definir el carácter sustantivo de la ciudadanía europea dado que todavía existen muchas dudas al respecto, sobre todo en el ámbito de los derechos fundamentales (Silveira, 2014).

Por un lado, como ya se ha mencionado, los europeos son cada vez más conscientes de su condición de ciudadanos de la Unión y, por otro, la UE toma cada vez más medidas para dar a conocer mejor los derechos de la ciudadanía (Comisión Europea, 2017a), fomentando la participación cívica de los jóvenes, entre quienes los medios de comunicación digitales tienen una gran repercusión.

El derecho a la Iniciativa Ciudadana Europea, hoy en día en el Reglamento (UE) 211/2011 del Parlamento Europeo y del Consejo, publicado en el Diario Oficial (OF, en inglés) L 65 de 11.3.2011, 1-22 (Alves, 2012; Gonçalves, 2016) fue introducido en el Tratado de Lisboa (artículo 11, (apartado 4 del Tratado de la Unión Europea (TEU) y apartado 4 del artículo 24 del Tratado de Funcionamiento de la Unión Europea (TFUE).

\section{Derecho de Iniciativa Ciudadana Europea}

El derecho a la Iniciativa Ciudadana Europea es un instrumento innovador de democracia participativa transnacional en vigor desde abril de 2012. Esta iniciativa permite que un millón de ciudadanos de la UE, de al menos siete Estados miembros, inviten a la Comisión Europea a presentar propuestas legislativas en ámbitos de competencia de la Unión Europea, como condiciones legal de admisibilidad. Además, deben referirse a cuestiones que son competencia de la Comisión para que este órgano pueda presentar una propuesta legislativa que permita la aplicación de los tratados. De este modo, se pretende que los ciudadanos participen activamente en el proceso de toma de decisiones europeo, proporcionándoles

2 Puede consultarse en https://participacao.parlamento.pt/initiatives/76. 
una forma indirecta de iniciativa legislativa. Hasta la fecha, más de seis millones de ciudadanos europeos han firmado una Iniciativa Ciudadana Europea. Hasta 2018 se presentaron 69 iniciativas de las cuales se registraron 48 por la Comisión Europea según el Comité de las Regiones (2018b), se rechazaron 17 solicitudes, 15 fueron retiradas y 25 no recibieron suficiente apoyo. Cabe señalar que un proceso de recogida de firmas, de 12 meses de duración, fue puesto en marcha por parte de sus organizadores en el que participó un millón de ciudadanos, de al menos una cuarta parte de los Estados miembros de la UE, en apoyo de la propuesta de Iniciativa Ciudadana Europea. Se trata de una invitación a la Comisión Europea para que presente propuestas legislativas, por supuesto dentro de sus ámbitos de competencia, y por lo tanto no puede ser contraria a los valores de la Unión. Por otra parte, en el plazo de uno a tres meses después del registro de la iniciativa, la Comisión Europea puede presentar propuestas legislativas, siempre en los ámbitos de su competencia, decidiendo si debe o no intervenir y explicando las razones de su posición, con conclusiones jurídicas y políticas (Comisión Europea, 2014b). Hasta la fecha no ha habido ninguna iniciativa plenamente exitosa; es decir, solo una ha dado lugar a un compromiso para una propuesta legislativa (Comité Económico y Social Europeo, 2018), aunque, en algunos casos, la Comisión Europea ha considerado el posicionamiento de la opinión pública sobre aspectos específicos. Pese a ello, es un instrumento de futuro de gran importancia democrática del que habrá que estar pendiente.

Desde el año 2012 y con el objetivo de llamar la atención tanto de los medios de comunicación como del público sobre la Iniciativa Ciudadana Europea en el sentido de dar una amplia visibilidad y sensibilizar sobre el tema (Comité Europeo de las Regiones, 2018b), el Comité Económico y Social Europeo (2018) ha creado un día conmemorativo anual que ya cuenta con siete ediciones.

La Comisión Europea considera que la Iniciativa Ciudadana Europea está “totalmente operativa” (2017e) pero reconoce que es posible mejorarla. Asimismo, el Parlamento Europeo (2015) ha seguido de cerca este mecanismo jurídico, tratando de mejorarlo para convertirlo en un instrumento de democracia participativa más accesible y fácil de utilizar para evitar barreras técnicas y burocráticas (Parlamento Europeo, 2017b) a su utilización. Y la Comisión Europea ya ha presentado una propuesta de modificación del Reglamento (Comisión Europea, 2017c) con medidas para facilitar la organización de la iniciativa ciudadana, facilitar el apoyo a las iniciativas y aumentar el impacto de las iniciativas que han tenido éxito. El proceso legislativo de modificación se está considerando con sumo cuidado porque refleja la preocupación por su impacto en el funcionamiento actual, el cual es la prioridad legislativa establecida por la Comisión Europea (2017b) para 2018. El Comité Europeo de las Regiones (2018b) afirma que, si este nuevo reglamento fracasa, será el final de la Iniciativa Ciudadana Europea.

La característica esencial del proceso legislativo europeo es que se trata de un proceso de negociación que requiere la participación de las instituciones europeas, los Estados miembros e incluso los ciudadanos (Marrana, 2012).

\section{Metodología de la investigación}

El conocimiento de carácter científico se basa en hechos y lenguajes propios y, siempre que es posible, la ciencia respalda sus axiomas teóricos con datos observables y repetibles. Según McMillan \& Schumacher (1997), el conocimiento científico dispone de estrategias y técnicas con el fin de garantizar la mayor precisión posible en los resultados que se obtienen. En 
cualquier investigación, la estrategia de investigación es una decisión básica ya que comunica tanto los resultados previstos de un estudio así cómo deben evaluarse los mismos. Las diferentes estrategias de investigación existentes no son incompatibles entre sí; sin embargo, hay que ser capaz de identificar algunas situaciones en las que una estrategia específica tiene clara ventaja sobre otras estrategias (Yin, 2009). En el caso que nos ocupa, además del análisis de alguna doctrina y más estrechamente de la documentación oficial de la Unión Europea, se ha seleccionado la metodología del estudio de caso. Según Yin (2009), en el estudio de caso el “cómo” y "por qué” son preguntas centrales sobre un conjunto de acontecimientos actuales sobre los que el investigador tiene poco o ningún control. Además, para Guba \& Lincoln (1994), el propósito del enfoque del estudio de caso es informar sobre los hechos tal como ocurrieron, describir situaciones o eventos, proporcionar conocimiento sobre el evento estudiado y probar o contrastar los efectos y relaciones presentes en el caso.

La investigación que presentamos se caracteriza por el análisis de un hecho en un entorno real, con el objetivo de aclarar, "el grado de utilización de la plataforma en las peticiones y la evolución de este mismo uso desde la primera vez que se utilizó por parte de distintas organizaciones y ciudadanos hasta el presente...”; así, la estrategia de investigación adoptada fue el estudio de caso.

Un paso clave en la planificación y realización de un estudio de caso es la definición de la unidad de análisis, es decir, la definición del objeto de estudio (Yin, 2009). Y, en este sentido, como se ha dicho, la unidad de análisis de la investigación es la plataforma que promueve la Iniciativa Ciudadana Europea (Comisión Europea, 2018). Como técnica de recogida de datos, se ha utilizado la información que proporciona la plataforma sobre su uso en diferentes momentos. Como estrategia principal para el análisis de datos, hemos utilizado el “análisis de comparación constante” (Glaser, 1978).

\section{Plataforma de la Iniciativa Ciudadana Europea - Estudio de caso}

La idoneidad de una plataforma digital para el funcionamiento y la puesta en marcha de la Iniciativa Ciudadana Europea (Comisión Europea, 2018) ha puesto la tecnología al servicio de la democracia y de los ciudadanos de toda la Unión, permitiéndoles acceder a un instrumento que de otro modo no tendría una incidencia directa.

Este es sólo uno de los ejemplos en los que se puede analizar este nuevo vínculo entre el uso de las nuevas tecnologías y la participación democrática, transformando tanto la realidad política como los fenómenos políticos (Marcos García, Alonso Muñoz y Casero-Ripollés, 2017). De hecho, el uso de plataformas en línea es una de las nuevas formas de participación política que Aguilera y Casero Ripollés denominan “extra representativa” en el sentido de que va más allá de los mecanismos institucionales establecidos (2018).

Las denominadas herramientas informáticas se mencionan en la documentación de la Unión Europea como un medio para reforzar los valores democráticos (Parlamento Europeo, 2018a) y es evidente que están al servicio de la construcción de una sociedad más fuerte, orientada hacia la integración.

De esta forma, esta transformación digital (Ferreira, et al., 2015) -que supone el uso de este instrumento de participación ciudadana a través de medios digitales- responde a los objetivos de transparencia que se persiguen en el ordenamiento jurídico de la Unión Europea, establecidos en el artículo 15, apartado 3, del TUE en concordancia con el artículo 42 de la 
Carta de los Derechos Fundamentales de la Unión Europea, con el fin de hacer visible la "huella legislativa" (Parlamento Europeo, 2018b) para la elaboración de cada estándar.

Este fenómeno se manifiesta en los medios sociales (una de las herramientas desarrolladas en el contexto de la web 2.0). Con la Web 2.0 cualquier usuario puede asumir un papel activo, siendo así el promotor del desarrollo de sus propios contenidos, a diferencia de la web 1.0 donde esa función sólo era posible para programadores especializados) como analizó Casero-Ripollés (2017).

Entre otras nuevas tecnologías, el acceso de los ciudadanos a una plataforma digital -que puede funcionar en cualquier dispositivo digital y en cualquier momento (Casero-Ripollés, 2018)- para expresar su apoyo a una iniciativa legislativa supone una gran novedad en cuanto a la participación ciudadana en el sistema político.

Para desarrollar nuestra investigación y responder a las preguntas de la investigación, se llevaron a cabo las siguientes tareas:

1. Recogida de datos - la información se obtuvo de la plataforma de la ICE;

a) Iniciativas exitosas

b) Iniciativas logradas

i. Retiradas

ii. Insuficiente apoyo

2. Análisis de la información y

3. Utilización de una herramienta para diseñar un gráfico con el fin de obtener un análisis comparativo de los resultados. Esta opción se justifica por el hecho de que la lectura y comparación de los resultados es más sencilla.

\subsection{Contexto}

La plataforma de la Iniciativa Ciudadana Europea (ICE) tal y como se ha mencionado, es una plataforma en línea que está a disposición de cualquier ciudadano u organización y que ofrece diversa información general, así como iniciativas abiertas, iniciativas exitosas (que son el objeto del presente estudio) y lanzamiento de la iniciativa (ver Fig. 1). 


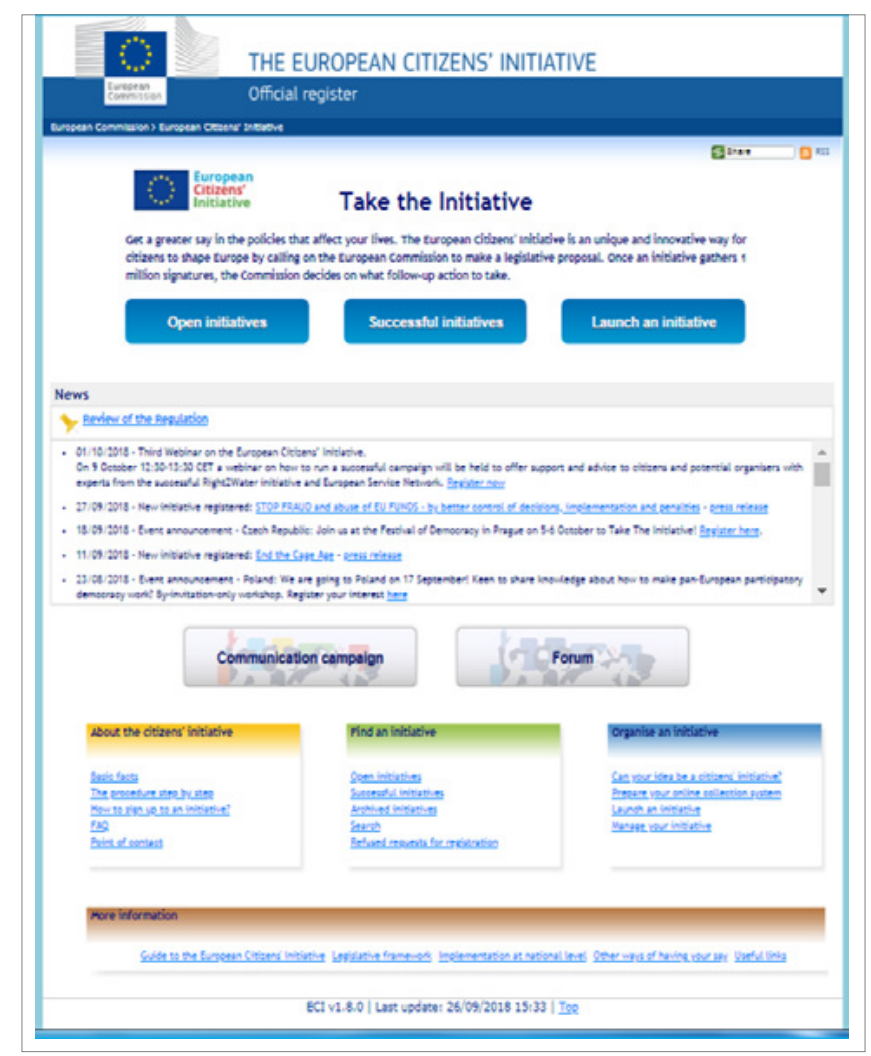

Figura 1. Plataforma ICE [Fuente: (Comisión Europea, 2018)].

\subsection{Recogida de datos}

Para analizar en qué medida se utiliza la plataforma en las peticiones y la evolución de este mismo uso desde su primera utilización por parte de organizaciones y ciudadanos hasta ahora, se ha utilizado la información disponible (1) "iniciativas exitosas” y (2) "iniciativas logradas" - (a) iniciativas retiradas y (b) iniciativas con apoyo insuficiente (véase la Fig. 2). 


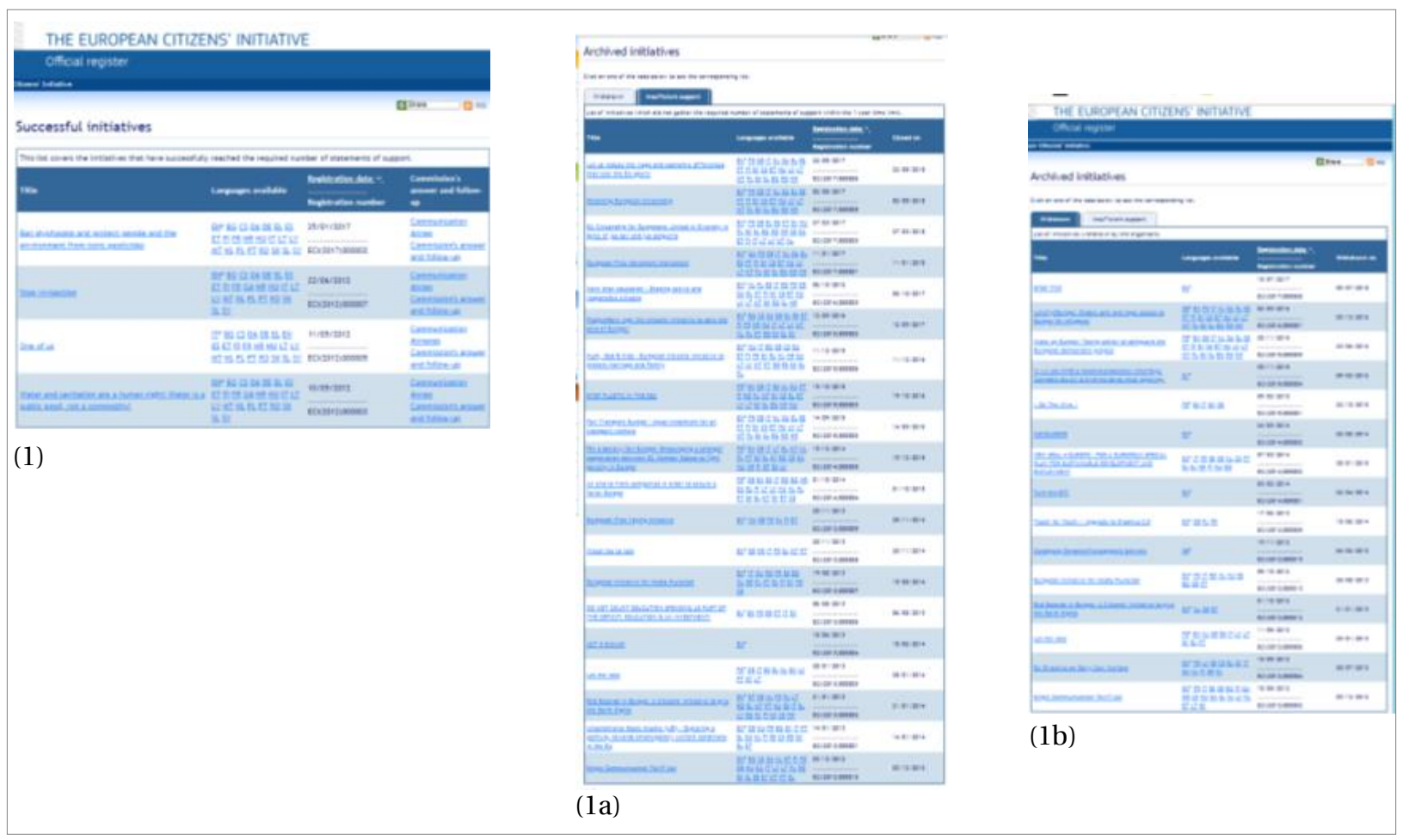

Figura 2. Iniciativas de la ICE [Fuente: (Comisión Europea, 2018)]].

La figura 3 muestra la lista de las iniciativas actualmente abiertas para la recogida de declaraciones de apoyo y aquellas otras para las que ya se ha cerrado la recogida, pero para las que la Comisión todavía no dispone de información sobre si los organizadores han gestionado o no la recogida del número requerido de declaraciones de apoyo. 


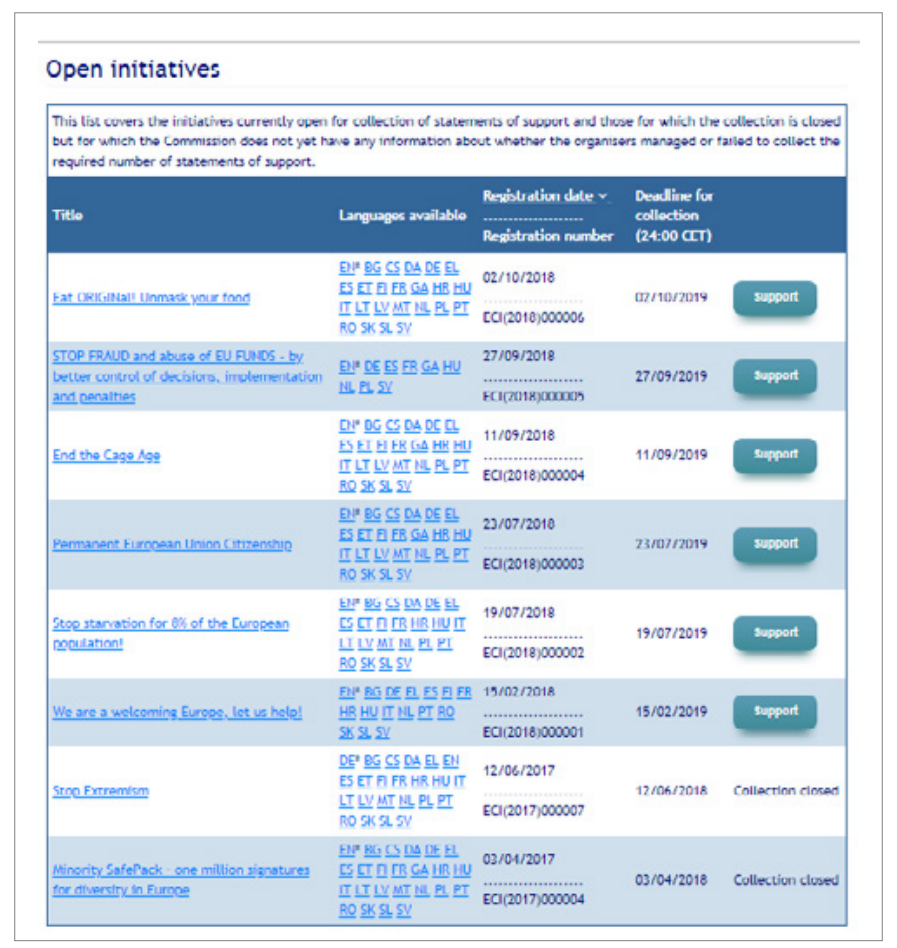

Figura 3. Iniciativas abiertas [Fuente: (Comisión Europea, 2018)]].

\subsection{Análisis de los datos}

A partir de la información proporcionada, podemos comprobar que la primera iniciativa tuvo lugar el 10/05/2012. Entre el 12/03/2012 y el 7/9/2018, los organizadores retiraron 15 iniciativas. Entre el 11/01/2013 y el 22/05/2018 hubo 26 iniciativas que no reunieron el número requerido de declaraciones de apoyo dentro del límite de un año, con lo cual fueron declaradas como "apoyo insuficiente". Entre el 10/05/2012 y el 25/01/2017 hubo 4 iniciativas que sí lograron alcanzar el número requerido de declaraciones de apoyo.

En la actualidad hay ocho (8) iniciativas en marcha; la primera se inició el 15/02/2018 y la última el 10/02/2018. Dentro de las peticiones en curso, hay dos (2) cerradas, pero aún no resueltas por la Comisión.

Las conclusiones presentadas se verán condicionadas, por supuesto, por el período de tiempo analizado. 


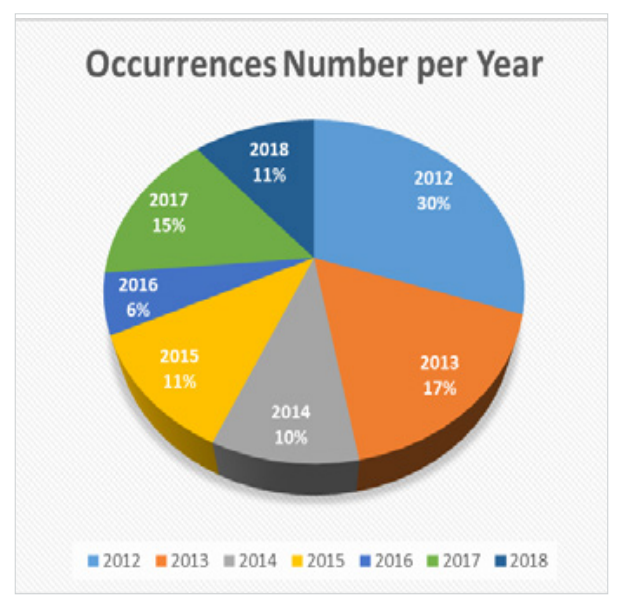

Gráfico 1. Porcentajes de ocurrencias, por año

Si analizamos el número de ocurrencias por año, independientemente de su éxito o no (ver Gráfico 1), se puede concluir que el año con mayor número fue 2012, con un porcentaje del 12\%. Y que durante los años siguientes no existe un patrón; es decir, entre 2013 y 2018 hay años en los que el número de ocurrencias disminuye, y otros en los que crecen, variando el porcentaje entre el $17 \%$ y el $11 \%$.

Del análisis de los datos se puede concluir que el uso de la plataforma no es significativo desde su creación hasta el presente. Finalmente, y en contra de lo esperado, el año con más éxito fue 2012.

Sin embargo y debido a la tendencia según la cual los ciudadanos cada vez más utilizan las nuevas tecnologías (cada vez están más digitalizados), el uso de la plataforma seguirá creciendo.

\section{Conclusiones}

La idea de fortalecer la democracia no limita o restringe la participación de los ciudadanos en los procedimientos relativos a la acción legislativa. Una mayor concienciación ciudadana puede dar a los ciudadanos la oportunidad de interactuar y participar en la construcción democrática. El nuevo concepto de hacer visible la "huella legislativa" se ve reforzado por el uso de los medios digitales.

Lo que entendemos por democracia sigue vinculado al ejercicio de las libertades individuales y al "diseño" de la estructura del Estado donde se reivindica la relevancia del ciudadano. El acercamiento de los ciudadanos a la construcción europea sigue en marcha. Esta interacción con los ciudadanos está siendo y debe ser reforzada tanto por la Unión Europea como por los Estados miembros, por el bien de la democracia, en la "construcción" del estado de derecho. La Iniciativa Ciudadana Europea es, en este sentido, más bien un vehículo; se la considera como una herramienta política para captar la atención de los ciudadanos, pero aún no ha dado los resultados necesarios para que el ciudadano de a pie la haga suya; 
además, existen aún dificultades técnicas ya identificadas y a las que las instituciones comunitarias ya han dado su apoyo con el fin de introducir mejoras mediante cambios en el régimen jurídico aplicable. Al igual que en la realidad portuguesa, todavía no se ha adoptado ninguna disposición legislativa, pero a nivel europeo se están tomando medidas para lograr una nueva legislación

El uso de la Iniciativa Ciudadana Europea ha aumentado mucho en el año de 2018 habiéndose registrado 6 nuevas iniciativas. De acuerdo con el diario oficial: Decisión (UE) 2018/262 de la Comisión, de 14 de febrero de 2018, diario oficial DO L 49 de 22.02.2018, 64-65, sobre la propuesta de iniciativa ciudadana “Somos una Europa acogedora, ¡Dejadnos ayudar!” Decisión (UE) 2018/517 de la Comisión, de 21 de marzo de 2018, DO L 84 de 28.3.2018, 25-26, “iAmigos británicos, quédense con nosotros en la UE!” Decisión de la Comisión 2018 / C 260/07 de 18 de julio de 2018, DO C 260 de 24.07.2018, 6-7, “¡Erradiquemos el hambre que aqueja al 8\% de la población europea!”. Decisión 2018 / C 264/05 de la Comisión, de 18 de julio de 2018, DO C 264 de 26.7.2018, 4-5, “Ciudadanía permanente de la Unión Europea”. Decisión (UE) 2018/1222 de la Comisión, de 5 de septiembre de 2018, DO L 226 de 10.9.2018, 7-8, “No más jaulas”. Decisión (UE) 2018/1304 de la Comisión, de 19 de septiembre de 2018, DO L 244 de 28.09.2018, 107-108, “¡Come ORIGINAL! Desenmascara tu comida”. Es interesante ver cómo el fenómeno de Brexit, con la salida del Reino Unido de la Unión Europea por el mecanismo del artículo 50 del Tratado de la Unión Europea, ha impulsado algunas iniciativas.

Existe un incentivo para que los ciudadanos europeos participen activamente en la política y en el futuro de Europa mediante plataformas digitales que permiten tanto la transparencia como la visibilidad. Sin embargo, la Iniciativa Ciudadana Europea, las peticiones debido a la iniciativa por parte de organizaciones o ciudadanos, aunque ha ido creciendo no puede considerarse aún una "herramienta" de gran utilidad. Esta situación deberá ser potenciada mediante acciones de publicidad. Por otro lado, se observa que la UE no es ajena a la era de la transformación digital, donde el uso de las Tecnologías de la Información (TI) son la esencia de las organizaciones modernas, en general; así, como ya se ha mencionado, se están produciendo cambios en este campo, creándose instrumentos apoyados por las tecnologías para promover una mayor cercanía e interés en la participación de los ciudadanos (muchos de los cuales ya están formados en los medios digitales) con los de la propia UE.

Todavía no es posible, dada la poca madurez del medio utilizado, sacar conclusiones globales sobre el cambio en la práctica de la participación política a través del uso de plataformas digitales como la creada para la Iniciativa Ciudadana Europea. La evolución del uso de dicha plataforma, tal y como se muestra en la figura 1, no es una evolución continua, es decir, se producen aumentos y disminuciones en el número de usos desde 2012 (fecha de creación) hasta la fecha analizada (2018). En este contexto, la presente investigación no se considera cerrada sino un trabajo en curso. La evolución de la plataforma se seguirá analizando en los trabajos a llevar a cabo en el futuro.

Las autoras consideran que se trata de una forma nueva y ventajosa de participación política, con una herramienta tecnológica que está a disposición de cada ciudadano, pero cuyas ventajas y desventajas aún no están suficientemente estudiadas. Para ello será preciso esperar algunos años para que el devenir de los acontecimientos históricos refuerce los datos. 


\section{Referencias bibliográficas}

Alves, D. R. (2012). A entrada em vigor do direito de iniciativa de cidadania europeia. Revista Jurídica, 15, 49-56. (Porto: Universidade Portucalense Infante D. Henrique.)

Aguilera, M. \& Casero Ripollés, A. (2018). ¿Tecnologías para la transformación? Los medios sociales ante el cambio político y social. Revista ICONO14 Revista Científica De Comunicación Y Tecnologías Emergentes, 16(1), 1-21. https://doi. org/10.7195/ril4.v16il.1162

Bonavides, P. (2001). Teoria constitucional da democracia participativa. Malheiros Editores.

Casero-Ripollés, A. (2018). "Research on political information and social media: key points and challenges for the future Investigación sobre información política y redes sociales: puntos clave y retos de futuro” El profesional de la información, 27, 964 - 974. https://doi.org/10.3145/epi.2018.sep.01

Casero-Ripollés, A. (2017). Producing political content for web 2.0: empowering citizens and vulnerable populations Producción de contenidos políticos, empoderamiento ciudadano y públicos vulnerables en la web 2.0. El profesional de la información, 26, 1, 13-19. https://doi.org/10.3145/epi.2017.ene.02

Chadwick, A. (2017). The Hybrid Media System: Politics and Power (Oxford Studies in Digital Politics) Oxford University Press. Counsil (2017). Conclusões 2017/C 62/02 do Conselho e dos Representantes dos Governos dos Estados-Membros, reunidos no Conselho, sobre a inclusão na diversidade a fim de alcançar uma educação de elevada qualidade para todos, JOUE C 62 de 25.02.2017, 3-7.

European Commission (2018). Relatório ao Parlamento Europeu e ao Conselho sobre a aplicação do Regulamento (UE) n. ${ }^{\circ} 211 / 2011$ do Parlamento Europeu e do Conselho de 16 de fevereiro de 2011 sobre a iniciativa de cidadania. Documento COM (2018) 157 final de 28.03.2018.

European Comission (2017a). EU Citizenship Report 2017 Strengthening Citizens' Rights in a Union of Democratic Change. Luxembourg: Publications Office of the European Union. doi:10.2838/51061.

European Commission (2017b). Comunicação ao Parlamento Europeu, ao Conselho, ao Comité Económico e Social Europeu e ao Comité das Regiões. Programa de Trabalho da Comissão para 2018. Programa para uma Europa mais unida, mais forte e mais democrática. Documento COM(2017) 650 final de 24.10.2017.

European Commission (2017c). Proposta de Regulamento do Parlamento Europeu e do Conselho sobre a iniciativa de cidadania europeia. Documento COM(2017) 482 final de 13.09.2017.

European Commission (2017d). Relatório da Comissão ao Conselho e ao Parlamento Europeu Relatório de avaliação ex post relativo ao programa «Direitos Fundamentais e Cidadania» (2007 2013). Documento COM(2017) 69 final de 13.02.2017. European Commission (2017e). Relatório ao Parlamento Europeu, ao Conselho, ao Comité Económico e Social Europeu e ao Comité das Regiões nos termos do artigo 25. ${ }^{\circ}$ do TFUE sobre os progressos rumo a uma efetiva cidadania da UE 20132016. Documento COM(2017) 32 final de 24.01.2017. 
European Commission (2017f). Relatório ao Parlamento Europeu, ao Conselho, ao Comité Económico e Social Europeu e ao Comité das Regiões Reforçar os direitos dos cidadãos numa União da mudança democrática Relatório de 2017 sobre a Cidadania da UE. Documento COM(2017) 30 final de 24.01.2017.

European Commission (2016). Comunicação ao Parlamento Europeu, ao Conselho, ao Comité Económico e Social Europeu e ao Comité das Regiões Proposta de um novo Consenso Europeu sobre o Desenvolvimento O nosso mundo, a nossa dignidade, o nosso futuro. Documento COM (2016) 740 final de 22.11.2013.

European Comission (2014a). Did you know? 10 EU rights at a glance. Luxembourg: Publications Office of the European Union. doi:10.2775/22234.

European Commission (2014b). Comunicação sobre a iniciativa europeia “A água e o saneamento são um direito humano! A água não é um bem comercial, mas um bem público!”. Documento COM(2014) 177 final de 19.03.2014

European Commission (2003). Comunicação ao Conselho e ao Parlamento Europeu sobre o artigo $7^{\circ}$ do Tratado da União Europeia. Respeito e promoção dos valores em que a União assenta. Documento COM(2003) 606 final de 15.10.2003.

European Commission (2002). Comunicação da Comissão sobre a melhoria do controlo da aplicação do direito comunitário. Documento COM(2002) 725 final de 11.12.2002.

European Commission (2001). Governança Europeia - Um Livro Branco 2001/C 287/01, JOUE C 287 de 12.10.2001, 1-29.

European Committee of the Regions (2018a). Parecer 2018/C 361/05 Reforçar a identidade europeia através da educação e da cultura, JOUE C 561 de 05.10.2018, 19-30.

European Committee of the Regions (2018b). Parecer 2018/C 247/ 10 sobre a Iniciativa de Cidadania Europeia, JOUE C 247 de 13.07.2018, 62-70.

European Committee of the Regions (2015). Resolução 2015/C 313/03 sobre o tema «Melhorar o funcionamento da União Europeia: O Tratado de Lisboa e mais além», JOUE C 313 de 22.09.2015, 9-11.

European Economic and Social Committee (2018). Parecer 2018/C 237/12 sobre a «Proposta de regulamento do Parlamento Europeu e do Conselho sobre a iniciativa de cidadania europeia», JOUE C 237 de 06.07.2018, 74-80.

European Parliament (2018a). Resolução 2018/C 337/04 de 12 de setembro de 2017 sobre a formação universitária contínua e à distância como parte da Estratégia europeia em prol da aprendizagem ao longo da vida, JOUE C 337 de 20.09.2018, 20-29. European Parliament (2018b). Resolução 2018/C 337/ 18 de 14 de setembro de 2017 sobre transparência, responsabilidade e integridade nas instituições da UE, JOUE C 337 de 20.09.2018, 120-130.

European Parliament (2018c). Resolução do Parlamento Europeu 2017/C 355/03, de 28 de outubro de 2015, sobre a Iniciativa de Cidadania Europeia, JOUE C 355 de 20.10.2011, 20.

European Parliament (2018d). Regulamento (UE) n. ${ }^{\circ}$ 211/2011 e do Conselho de 16 de Fevereiro de 2011 sobre a iniciativa de cidadania, JOUE L 65 de 11.03.2011, 1-22.

European Parliament (2017a). Resolução 2017/C 408/02 de 2 de abril de 2014, sobre a revisão intercalar do Programa de Estocolmo, JOUE C 408 de 30.11.2017, 8-20. 
European Parliament (2017b). Resolução 2017/C 378/25, de 13 de março de 2014, sobre a aplicação do Tratado de Lisboa no que respeita ao Parlamento Europeu, JOUE C 378 de 09.11.2017, 218-226.

European Parliament (2015). Resolução do Parlamento Europeu 2010/C 212 E/14, JOUE C 212 E de 05.08.2010, pp. 99 a 105, e Resolução do Parlamento Europeu, de 28 de outubro de 2015, sobre a Iniciativa de Cidadania Europeia, P8_TA(2015)0382. Ferreira, M. J., Moreira, F. \& Seruca, I. (2015). Social Business: A Way to Promote Organizational Transformation. International Journal of Information System Modeling and Design (IJISMD). 6(4), 57-81. DOI: 10.4018/IJISMD.2015100104 (ISSN 1947-8186; eISSN 1947-8194).

Gil de Zúñiga, H.; Huber, B.; Strauß, N. (2018). “Social media and democracy”. El profesional de la información, 27, 6, 11721180. https://doi.org/10.3145/epi.2018.nov.01

Glaser, B. (1978). Theoretical sensitivity. Mill Vally: The Sociology Press.

Gonçalves, R. M. (2016). Novas alternativas ao exercício da democracia tradicional: Ademocracia participativa. Revista Jurídica, 3(44), 426-437. Curitiba. doi: 10.6084/m9.figshare.4610839

Guba, E., \& Lincoln, Y. (1994). Competing paradigms in qualitative research. In N. K. Denzin \& Y. S. Lincoln (Eds.), Handbook of qualitative research (pp. 105-117). SAGE Publications.

Maior, M. S. (1998). O direito de acção popular na Constituição da República Portuguesa. Documentação e Direito Comparado, 75/76. Retrieved from http://www.gddc.pt/actividade-editorial/pdfs-publicacoes/7576-g.pdf

Marcos García, S.; Alonso Muñoz, L. \& Casero-Ripollés, A. (2017). Usos ciudadanos de Twitter en eventos políticos relevantes. La \#SesiónDeInvestidura de Pedro Sánchez. Comunicación y Hombre Revista interdisciplinar de Ciencias de la Comunicación y Humanidades, 13, 25-49. Madrid: Editorial Universidad Francisco de Vitoria.

Marrana, R. M. (2012). O acesso à informação no quadro do funcionamento da União Europeia. Revista de Ciências Empresariais e Jurídicas, 21, 7-42.

McMillan, J., \& Schumacher, S. (1997). Research in Education: a conceptual introduction. New York: Longman.

Moury, C. (2016). A democracia na Europa. Fundação Francisco Manuel dos Santos.

Murray A. (2016). Information Technology Law: The Law and Society. Oxford: Oxford University Press.

Pais, S. O. (2010). O Tratado de Lisboa e a renovação das instituições da União Europeia. Cadernos O Direito O Tratado de Lisboa, 5, 319-350.

Silva, M. M. M., \& Alves, D. R. (2016). Noções de Direito Constitucional e Ciência Política. 3rd. ed. Lisboa: Rei dos Livros.

Silveira, A. (2014). Do âmbito de aplicação da Carta dos Direitos Fundamentais da União Europeia: Recai ou não recai? - Eis a questão! Revista Julgar digital, 22, 179-209.

Silveira, A., \& Canotilho, M. (2013). Carta dos Direitos Fundamentais da União Europeia: Comentada. Coimbra: Almedina. Schuelke-Leech, B.-A. (2018). A model for understanding the orders of magnitude of disruptive technologies. Technological Forecasting \& Social Change, V. 129, pp. 261-274.

Yin, R. K. (2009). Case study research: Design and methods (4th Ed.). Thousand Oaks, CA: Sage. 
